\title{
Investigation of a Method for RF Circuits Analysis Based on Electromagnetic Topology
}

\author{
Yoon-Mi Park ${ }^{\dagger}$, Young-Seek Chung*, Changyul Cheon** and Hyun-Kyo Jung***
}

\begin{abstract}
In this paper, electromagnetic topology (EMT) was used to analyze the electromagnetic compatibility (EMC) of RF circuits including passive and active components. It is difficult to obtain usable results for problems relating to electromagnetic coupling in complex systems when using conventional numerical or experimental methods. Thus it is necessary to find a new methodology for analyzing EMC problems in complicated electromagnetic environments. In order to consider the nonlinear characteristics of active components, a SPICE diode model was used. A power detector circuit and the receiver circuit of a radio control (RC) car were analyzed and experimented in order to verify the validity of this method.
\end{abstract}

Keywords: BLT equation, EMI/EMC, EMT, nonlinear circuit

\section{Introduction}

Due to the recent rapid evolution of electronic technology, modern electric systems have become increasingly complicated. As a result, concern with the electromagnetic coupling phenomena by aperture and transmission line coupling has been growing for the past several years as they often cause significant problems that have a negative impact on electrical systems.

There is a limit to the use of conventional methods to analyze the EMC problems of complex circuits in enclosures such as computer mother boards owing to the difficulty of modeling the system [1]. The full wave analysis techniques used to analyze complex electronic systems are relatively accurate but require long computation times, detailed mesh generation and large amounts of data [2]. On the other hand, simulations based on circuit analysis techniques require short computation times but do not consider the external electromagnetic field coupling phenomenon and, therefore, analyze the phenomenon with low accuracy. Furthermore, the target circuit consists of various components as passive, active components. Because active components have nonlinear characteristics, determining the interaction characteristics of the components is difficult. Hence it is necessary to find a new methodology for analyzing EMC problems in complicated electromagnetic environments.

Electromagnetic topology (EMT) introduced by C. E. Baum in the 1980's is a simulation approach to analyze the electromagnetic interference/electromagnetic compatibility

$\dagger$ Corresponding Author : School of Electrical Engineering and Computer Sciences, Seoul National University, Korea.(iamyum00@snu.ac.kr)

* Department of Wireless Communications Engineering, Kwangwoon University Korea.( yschung@kw.ac.kr)

** School of Electrical and Computer Engineering, University of Seoul, Korea.( changyul@uos.ac.kr)

*** School of Electrical Engineering and Computer Sciences, Seoul National University, Korea. (hkjung@snu.ac.kr)

Received 25 July 2008; Accepted 4 August 2009
(EMI/EMC) phenomena. And, the EMT also provides a structured way to represent the electromagnetic interaction process in large electrical systems. In a topological network, it is possible to divide an entire system into smaller independent volumes that interact with each other through a systemic topological diagram. The interaction phenomena of the system are described by relationships between incoming and outgoing waves from each volume, namely the scattering parameter. Therefore, the EMT requires shorter computation times and its accuracy is validated at high frequency. Furthermore, it is possible for the EMT to combine a full wave analysis with a circuit analysis technique. In a previous paper, a method based on the EMT for analyzing RF circuits including only passive components was proposed [3]. Very few attempts have been made at the analysis of a circuit by EMT. However, the method has a limit to analyze nonlinear circuits.

In this paper, a simulation based on EMT is proposed to analyze complicated RF circuits including passive and active components. The results from the proposed method match well with reference data and responses predictions of the system were possible.

\section{Proposed Method}

\subsection{Analysis method based on EMT}

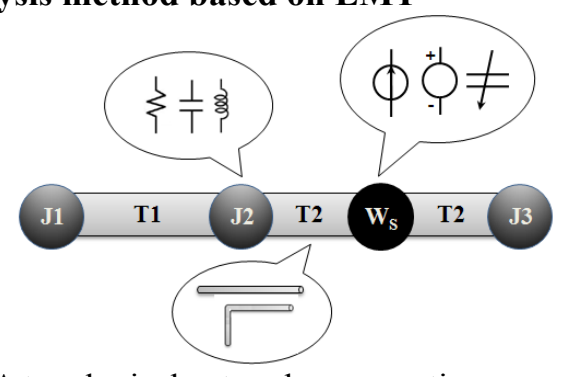

Fig. 1. A topological network representing a general circuit model including several components and sources 
EMT is a helpful technique for assessing EMC problems when analyzing complicated electric systems using volume decomposition. The basic principles of the EMT involve dividing the complex structure of a target system into a set of smaller volumes, characterizing the smaller volumes, and finally reconstructing the whole target system. The solution for the whole system can be determined using the Baum-Liu-Tesche (BLT) equation which reconstructs the divided volumes. The BLT equation describes voltage and current responses in a network of transmission lines. Fig. 1 shows the topological network model consisting of junctions, tubes, and sources.

The junctions indicate n-port devices in the circuit, such as resistors, inductors, capacitors and diodes, and represent the intersection and termination of a transmission line in the network. The tubes represent the connecting lines between junctions. Scattering parameters are used in the BLT equation, to describe the characteristics of junctions and tubes and can be obtained through numerical techniques such as experimentation, simulation or manufacturer specifications. Coupling between sources in the circuit is expressed as the equivalent generators applied to the tubes [3].

Fig. 2 shows incident and reflected waves at the (a) junctions and (b) tubes. In this approach, the scattering parameters are described by the equations (1) and (2). $W_{i}(0)$ denotes a reflected wave, $W j(L)$ denotes an incident wave, $S i j$ is the scattering parameter at the junction, $\Gamma$ is the scattering parameter at the tube and $W_{s i}$ is the equivalent source.

$$
\begin{gathered}
W_{i}(0)=S_{i j} W_{j}(L) \\
W_{i}(L)=\Gamma \times W_{i}(0)+W_{s i}
\end{gathered}
$$

The BLT equation that takes the coupling on the entire network into account is described by Equations (3-5) [4].

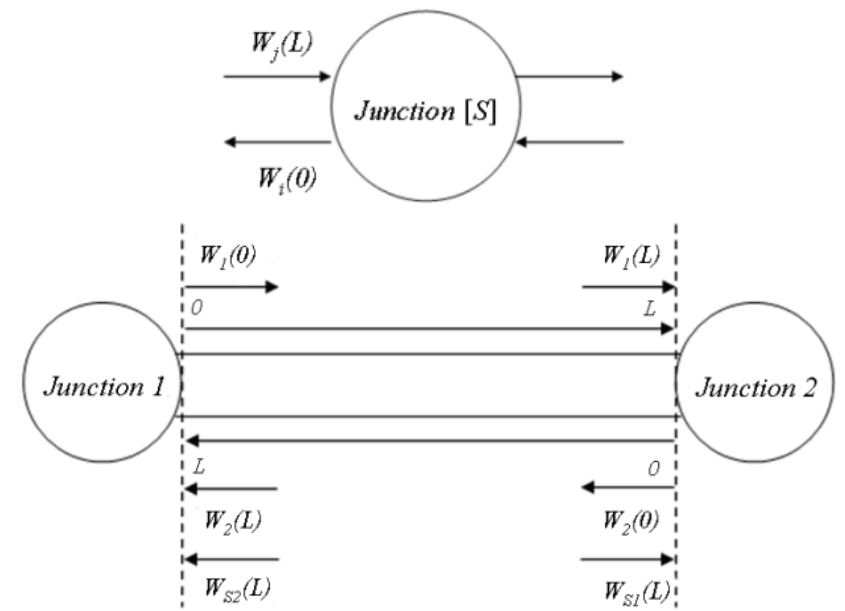

Fig. 2. Wave representation in terms of two-port network at the (a) junction and (b) tube

$$
\begin{gathered}
{[W(0)]=([I]-[S] \times[\Gamma])^{-1} \times[S] \times\left[W_{S}\right]} \\
{[W(L)]=[\Gamma] \times[W(0)]+\left[W_{S}\right]} \\
{\left[W_{\text {total }}\right]=[W(0)]+[W(L)]}
\end{gathered}
$$

The BLT equation consists of the propagation supermatrix $[\Gamma]$, the scattering supermatrix $[S]$, supervector $\left[W_{S}\right]$, the incident wave matrix $[W(L)]$ and the reflected wave matrix $[W(0)]$. These matrices can be determined by reconstituting the scattering parameters of the smaller volumes. With this method, we can obtain voltage and current responses at each junction. The BLT equation enables all connections and couplings on the topology network to be described.

\subsection{Active Component Modeling}

In topological analysis, the scattering parameters for components that compose the overall circuits can be obtained through various methods. A method for circuits including passive components was proposed in a previous paper [3]. However active components have nonlinear characteristics, thus there is a limit to the use of conventional techniques.

In this paper, a new diode SPICE model is proposed to obtain scattering parameters. The SPICE diode model is shown in Fig. 3. The parameters used in the SPICE model are from $600 \mathrm{MHz}$ to $700 \mathrm{MHz}$. Dynamic behaviors are modeled by the nonlinear capacitor $\mathrm{C}_{\mathrm{D}}$ and the series resistance $R_{S}$ represents the parasitic resistance. In this model, $i_{D}$ is diode current and $v_{D}$ is diode voltage [5].

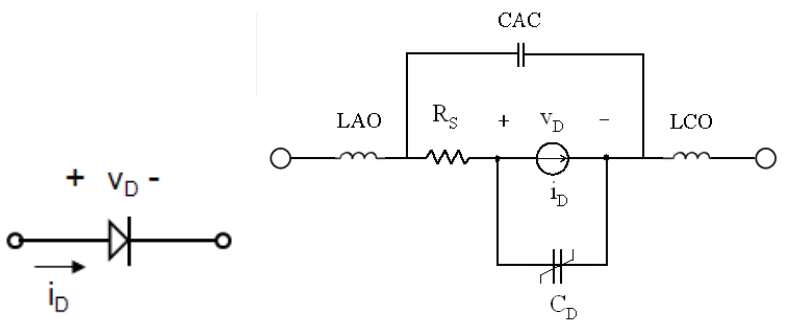

Fig. 3. SPICE model including the parasitic package components of the diode

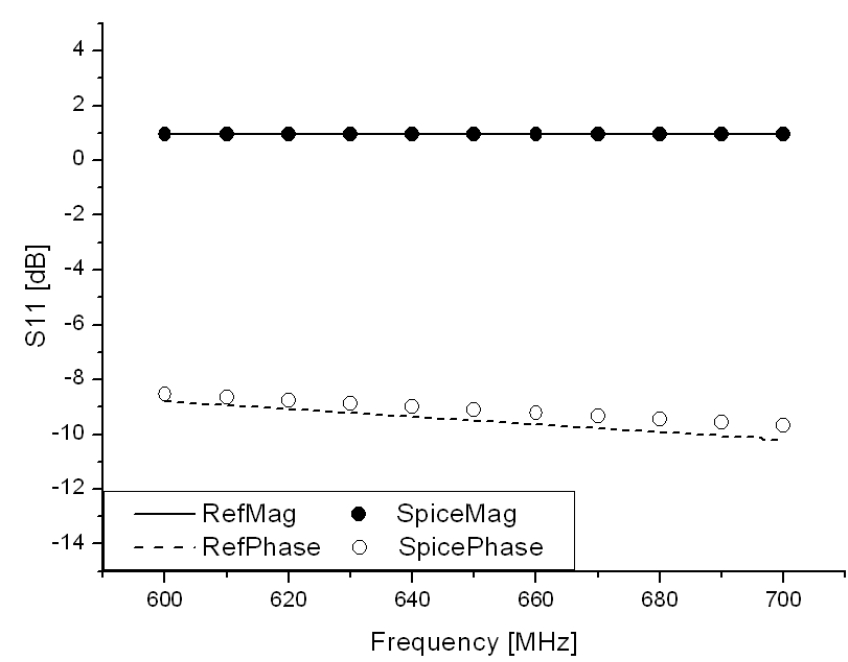

Fig. 4. Scattering parameters for the diode model, BAT62$02 \mathrm{~W}$

In order to observe the effect of various imperfections 
on device operation, parasitic package components, such as $\mathrm{LAO}, \mathrm{CAC}$, and LCO, are put in the SPICE model around the chip diode. The SPICE diode model replaces the diode as a junction in the topology diagram.

Fig. 4 shows the scattering parameters for the diode model, BAT 62-02W. In Fig. 4, a solid line is the magnitude of manufacturer specifications, a dotted line is the phase of manufacturer specifications, solid dots the magnitude of the SPICE model, and open dots the phase of the SPICE model. As Fig. 4 indicates, the scattering parameters from the SPICE model agree with the manufacturer specifications and the validity of the method is verified.

\section{Numerical Simulation and Results}

\subsection{Power Detector Circuit Model}

In order to demonstrate the proposed method, we considered a coplanar waveguide (CPW) circuit with a Low Noise Amplifier (LNA), diode and several R, L, and C components. The used LNA was MGA-87563 (Agilent Technologies) and the diode was BAT 62-02W (Infineon Technologies). In this paper, the analysis model was previously simulated and the result compared to measurement results.

Fig. 5 is a schematic of the analysis model and Fig. 6 shows the fabricated model and a topology diagram. The circuit consists of a chip LNA, diode and several RLC components. Inductors and capacitors are placed to match the input and output resistance to $50 \Omega$ in the schematic.

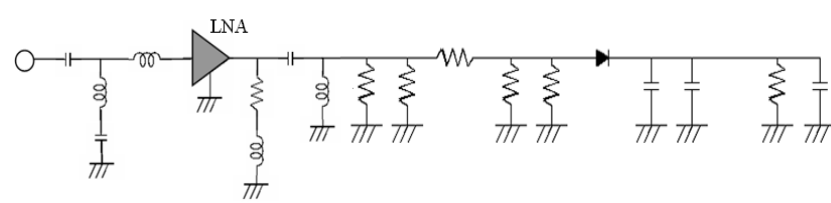

Fig. 5. Schematic of the analysis model including LNA, diode and several R, L, C components

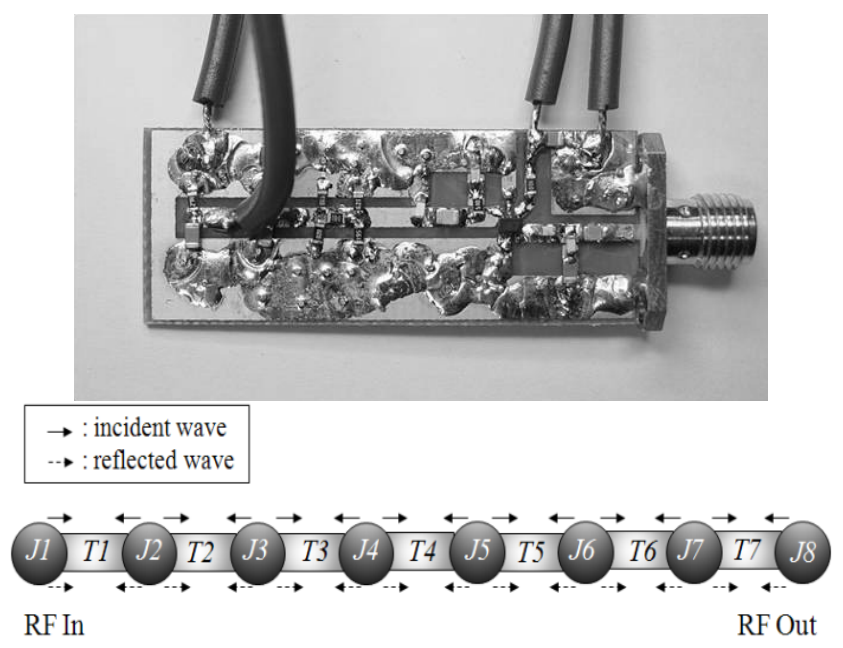

Fig. 6. The fabricated power detector circuit model and topology diagram representing the circuit model

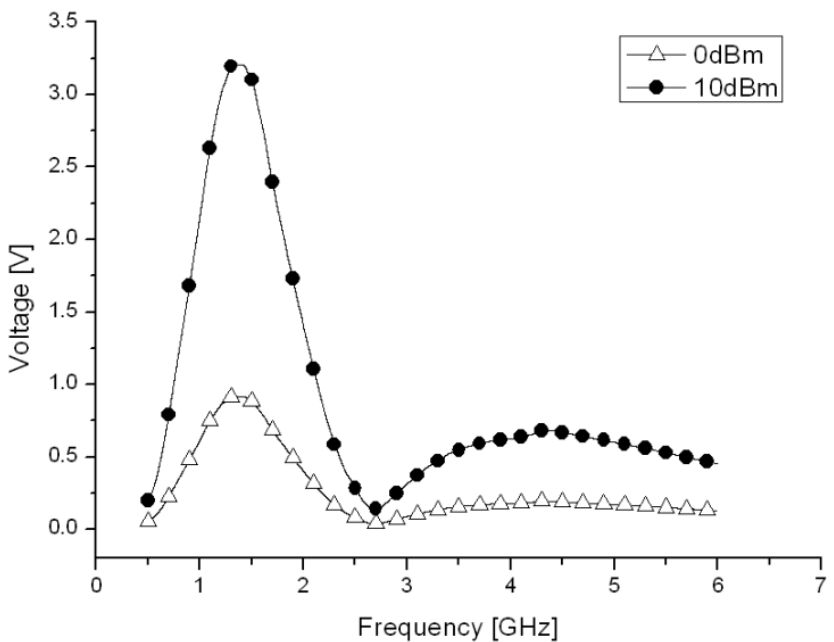

Fig. 7. The RF input voltage response at the LNA with input power set to $0 \mathrm{dBm}$ and $10 \mathrm{dBm}$ by the EMT

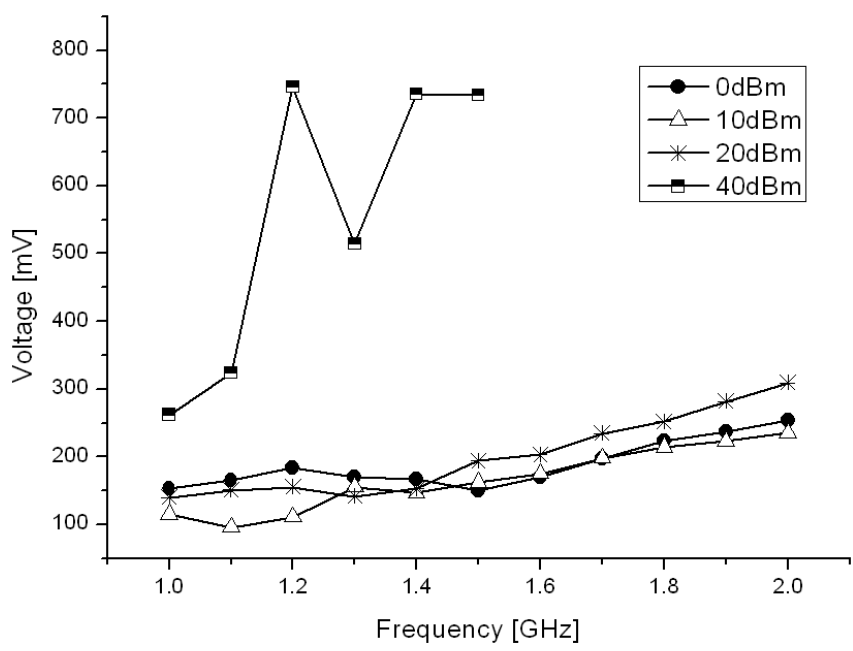

Fig. 8. The diode input voltage of the power detector circuit by experimentation with input power set to $0 \mathrm{dBm}, 10 \mathrm{dBm}, 20 \mathrm{dBm}$ and $40 \mathrm{dBm}$

In an EMT-based simulation, the interaction mechanism can be described as shown in Fig. 6. The topological approach analyzes electromagnetic coupling interactions at the junctions and tubes. In order to analyze the electromagnetic coupling interactions at junctions, the scattering parameters for circuit components were obtained from data tables and simulation. Through the BLT equation, the voltage and current response at the junctions can be obtained.

Fig. 7 shows the predicted input voltage response of the LNA by the EMT with input power set to $0 \mathrm{dBm}$ and $10 \mathrm{dBm}$. When the input power was $0 \mathrm{dBm}$, the RF input voltage to the ground was approximately $1 \mathrm{~V}$ at $1.5 \mathrm{GHz}$. When the input power was $10 \mathrm{dBm}$, the RF input voltage to the ground was above $1 \mathrm{~V}$ between $0.8 \mathrm{GHz}$ and $2.2 \mathrm{GHz}$. Since the maximum RF input voltage to the ground of the LNA was $1 \mathrm{~V}$ according to manufacturer specifications, the presence of an obstacle to the LNA was predicted in the case of $10 \mathrm{dBm}$ input power, which was verified through experimental results. 
Fig. 8 shows diode input voltage results by experimentation with input power set to $0 \sim 40 \mathrm{dBm}$. When the input power was above $10 \mathrm{dBm}$, the diode input voltage was less than the diode input voltage when the input power was $0 \mathrm{dBm}$. Specifically, the input power became $40 \mathrm{dBm}$ then the input voltage could not be measured because the LNA was physically destroyed at $1.5 \mathrm{GHz}$. It is found from the results that malfunction was generated since the input power was above $10 \mathrm{dBm}$ and these results were the same as the simulation results. Through this method, it is possible to predict the magnitude of the EMI source to disturb the performance of the circuit.

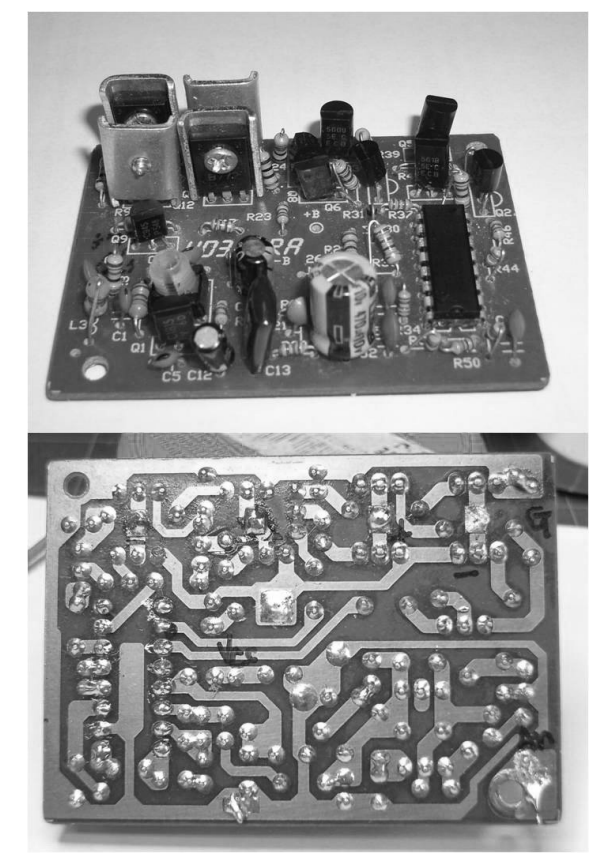

(a)

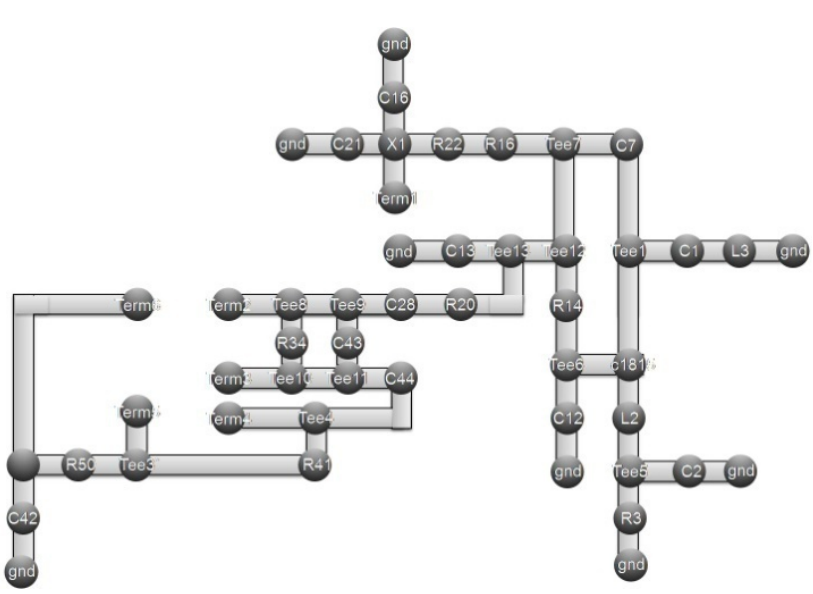

(b)

Fig. 9. (a) Top and bottom views of the $\mathrm{RC}$ receiver circuit model (b) topology diagram

\subsection{Application Model}

EMT was applied for the more complex model. Fig. 9 is the receiver circuit of a radio control (RC) car, Fig. 9(a) shows the top and bottom views of the physical model and Fig. 9(b) shows the topology diagram. When an RF voltage source is applied at the receiving antennas, the transmission path from the antennas to the important IC pin can be described through the EMT. The EMT results for the RC car are shown in Fig. 10 where the solid dots represent the results of the EMT, and the open dots the results of the commercial simulation tool. The simulation and the EMT results were similar within a range from 1 to $10 \mathrm{GHz}$. The results indicated that the source applied at the receiving antennas was delivered to the IC well at approximately 3, 6 and $9 \mathrm{GHz}$ and it was for this reason that internal circuit resonance was generated at 3,6 and $9 \mathrm{GHz}$.

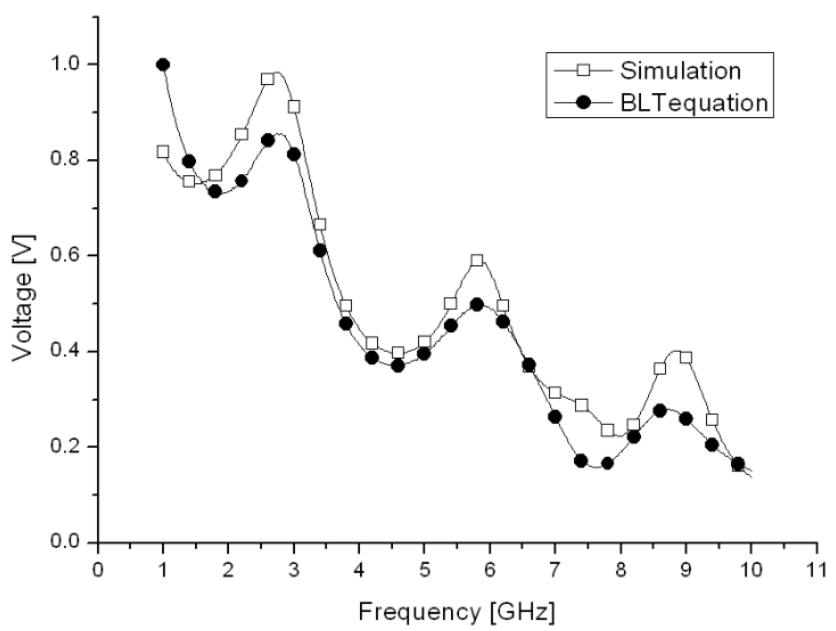

Fig. 10. Input voltage response of the $\mathrm{IC}$ in the $\mathrm{RC}$ receiver circuit

\section{Conclusions}

In this paper, RF circuits with active components were analyzed by EMT. In order to accommodate the nonlinear behavior of the diode, the SPICE diode model was applied to the EMT method. The results from topological modeling were in agreement with simulation and measured results. As a result, it is possible to predict the frequency response to disturb the performance of circuit. In this research, a circuit with an internal voltage source was studied, and from now on research related to external field coupling by EMT will be needed in order to apply the method to more practical issues.

\section{References}

[1] Zhou Dong-fang, Lin Jing-yu, Ren Jing-pu, Zhang De-wei, Yu Dao-jie, Niu Zhong-xia, "The Application of Electromagnetic Topology in the Analysis of HPM Effects on System", 6th International Symposium on Antennas, Propagation and EM Theory, 2003. Proceedings. 2003, 28 Oct.1 Nov. 2003, pp. 630-633.

[2] Phumin Kirawanich, Nakka S. Kranthi, and N. E. Islam, "Modeling External Interference on Systems 
Using Electromagnetic Topology Technique", International Symposium on Electromagnetic Compatibility 2004, Aug. 9-13 2004, p. 804.

[3] Yoon-Mi Park, Jung-Yub Lee, Sehoon Hwang, HyunKyo Jung, Young-Seek Chung and Hyeong-Seok Kim, "Analysis of Printed Circuit Boards Based on Electromagnetic Topology", Journal of Japan Society of Applied Electromagnetics and Mechanics, Vol. 15, No. 3, 2007.

[4] J. P. Parmantier, X. Ferrieres, S. Bertuol and C. E. Baum, "Optimization of the BLT Equation Based on a Sparse Gaussean Elimination," EMC International Zurich Symposium \& Technical Exhibition on Electromagnetic Compatibility Zurich, Feb. 16-18, 1999.

[5] Adel S. Sedra, K. Smith, Microelectronic Circuits, Oxford University Press, New York, 1998.

[6] F. M. Tesche, and C. M. Butler, "On the Addition of EM Field Propagation and Coupling Effects in the BLT Equation", Interaction Notes 588, Kirtland, Dec., 2003.

[7] F. M. Tesche, J. M. Keen, and C. M. Butler, "Examples of the Use of the BLT Equation for EM Field Propagation and Coupling Calculations," Interaction Notes 591, Kirtland, June, 2004.

[8] F. M. Tesche, M. Ianoz, and T. Karlsson, EMC, Analysis Methods and Computational Models, Wiley, New York, 1997.

[9] Jan Carlsson, Torbjörn Karlsson and Göran Undén, "EMEC-An EM Simulator Based on Topology", IEEE Transactions on Electromagnetic Compatibility, Vol. 46, No. 3, Aug. 2004.

[10] Jean Philippe Parmantier and Pierre Degauque, "Topology Based Modelling of very Large Systems", Modern Radio Science, Oxford U. Press, pp. 151-177, 1996.

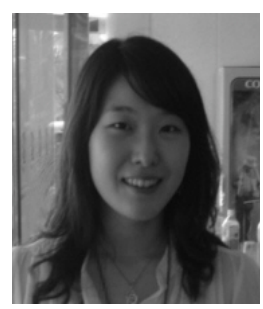

Yoon-Mi Park received her B.S. in Electrical Engineering at Chungnam National University in 2004. From 2004 she has studied for her M.S. and $\mathrm{Ph} . \mathrm{D}$. degrees at the School of Electrical Engineering and Computer Sciences, Seoul National University of Korea. Her research interests are numerical analysis of microwave, High Power Electromagnetics (HPEM) and Electromagnetic Interference/ Electromagnetic Compatibility (EMI/EMC) using FDTD, FEM and MoM.

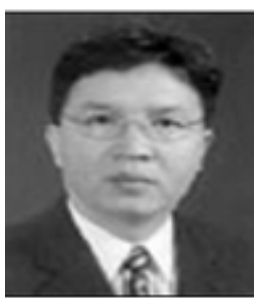

Young-Seek Chung received his B.S., M.S., and Ph.D. degrees in electrical engineering from Seoul National University, Seoul, Korea, in 1989, 1991, and 2000, respectively. From 1991 to 1996, he was with the Living System Laboratory, LG Electronics. From 1998 to 2000, he was a Teaching Assistant of electrical engineering with Seoul National University. From 2001 to 2002, he was with Syracuse University, Syracuse, NY. From 2003 to 2005, he has been a faculty member with the Department of Communication Engineering, Myongji University, Kyunggi, Korea. He is currently a Professor of the Department of Wireless Communications Engineering, Kwangwoon University, Seoul, Korea. His current interests are numerical analysis and inverse scattering using time-domain techniques.

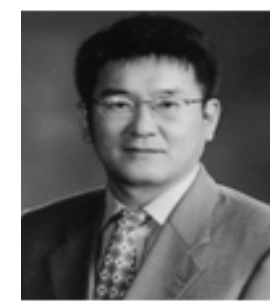

Changyul Cheon received his B.S. and M.S. degrees in electrical engineering from Seoul National University, Seoul, Korea in 1983 and 1985, respectively, and a Ph.D. degree in electrical engineering from the University of Michigan at Ann Arbor in 1992. From 1992 to 1995, he was with the Department of Electrical Engineering, Kangwon National University, Chuncheon, Korea, as an Assistant Professor. $\mathrm{He}$ is currently a professor of Electrical Engineering at the University of Seoul (UoS), Seoul, Korea. His group at UoS is currently involved with the design and analysis of microwave and millimeter-wave passive devices using FEM, FDTD, and MoM techniques. He is also interested in microwave sensors for biological applications.

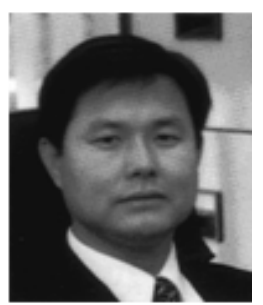

Hyun-Kyo Jung received his B.S., M.S., and Ph.D. degrees in electrical engineering from Seoul National University, Seoul, Korea, in 1979, 1981, and 1984, respectively. From 1985 to 1994, he was a member of the faculty at Kangwon National University. From 1987 to 1989 , he was with the Polytechnic University of Brooklyn, Brooklyn, NY. From 1999 to 2000, he was a Visiting Professor with the University of California at Berkeley. He is currently a Professor with the School of Electrical Engineering and Computer Science/Electrical Engineering, Seoul National University. His research interests are various fields of the analysis and the design of electric machines and the numerical analysis of microwave, HPEM, EMI/EMC and electrical systems, especially with the FEM. 